Schmerz 2011 $25: 613-616$

DOI 10.1007/s00482-011-1118-0

(c) Deutsche Gesellschaft zum Studium des Schmerzes. Published by Springer-Verlag all rights reserved 2011

\section{W. Koppert}

Klinik für Anästhesiologie und Intensivmedizin, Medizinische Hochschule Hannover
Auf der Mitgliederversammlung des zurückliegenden Schmerzkongresses in Mannheim wurde mit überwältigender Mehrheit eine Neuausrichtung der Deutschen Gesellschaft zum Studium des Schmerzes (DGSS) beschlossen. Wie bereits in der August-Ausgabe der „President's Corner" skizziert, wollen wir damit eine stärkere Präsenz erreichen, wenn es in Deutschland um das Thema Schmerz geht - nicht nur in der Wissenschaft, sondern auch in der Politik. Und wir wollen erreichen, dass viel mehr von dem Wissen, das die Forschung erarbeitet, in der täglichen Praxis und beim Patienten ankommt. Damit wollen wir noch mehr unseren Zielen gerecht werden, die interdisziplinäre Forschung, Diagnostik und Therapie von Schmerzen zu fördern.

Wir sehen diese Neuausrichtung dabei als konsequente Fortführung eines Wegs, der in den 7oer-Jahren begann und den ich Ihnen an dieser Stelle noch einmal in Erinnerung rufen möchte. Ohne die vielen Menschen, die als Pioniere der Schmerzforschung die Entwicklung der DGSS geprägt haben, stünden wir heute nicht dort, wo wir sind.

\section{Meilensteine}

Der 8. September 1975 war sicher ein Meilenstein. Damals wurde auf dem ersten World Congress on Pain in Florenz die Gesellschaft zum Studium des Schmerzes für Deutschland, Österreich und die Schweiz, kurz GesDÖS, gegründet. Damals hatten sich 31 Schmerzpioniere aufgemacht, um den Schmerz als eigenständige Erkrankung zu etablieren. Dies sollte ein langer Weg werden, wie wir heute wissen.

\title{
Aus der Deutschen Gesellschaft zum Studium des Schmerzes (DGSS) wird die Deutsche Schmerzgesellschaft
}

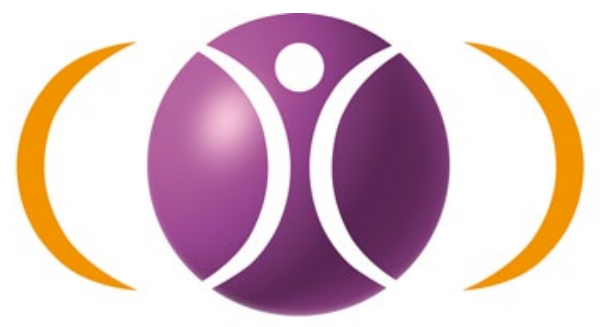

\section{Deuische Schmerzgesellschaft e.V.}

Schon früh kristallisierten sich 6 Arbeitsfelder heraus:

1. die Verbesserung der Ausbildung aller an der Schmerztherapie beteiligten Berufsgruppen;

2. die Anerkennung der Schmerztherapie als eigenes ärztliches Fachgebiet;

3. die Entwicklung von Maßnahmen zur Qualitätssicherung;

4. die Förderung des wissenschaftlichen Nachwuchses;

5. die Formulierung ethischer und politischer Forderungen und

6. die Information der Öffentlichkeit über neue wissenschaftliche Erkenntnisse.

\section{Ausbildung}

Zu Beginn unserer Arbeit waren Schmerzen sowie deren Diagnostik und Therapie nicht Gegenstand des Medizinstudiums. Schritt für Schritt haben wir zu einer Reform der universitären Ausbildung beigetragen. Wir entwickelten Lehreinheiten über Schmerz und Schmerztherapie im Medizinstudium. Doch auch in der aktuell gültigen Fassung der Approbationsordnung für Ärzte kommt die Schmerztherapie noch nicht vor. Zwar unterrichten die meisten der 34 medizinischen Fakultäten mittlerweile Schmerztherapie, es gibt aber immer noch Ärzte, die in ihrem Studium nichts über Schmerzen lernen. Um auch hier Abhilfe zu schaffen, haben wir ein „Kerncurriculum Schmerztherapie“ entwickelt, das in $14 \mathrm{~h}$ das Schmerzwissen für jeden angehenden Arzt zusammenfasst. Dies wird weiterhin ein zentraler Bereich unserer Arbeit sein, der aktuelle Referentenentwurf zur Änderung der Approbationsordnung lässt hier hoffen.

\section{Das Fachgebiet Schmerz}

In Bezug auf diesen Punkt haben wir bereits 1996 ein Ziel erreicht, für das wir zuvor ebenso lange gekämpft hatten: die Schaffung einer Zusatzbezeichnung „Schmerztherapie“. Gemeinsam mit dem damaligen Schmerztherapeutischen Kolloquium (STK), der heutigen Deutschen Gesellschaft für Schmerztherapie (DGS), hatten wir zunächst Standards und Ausbildungsinhalte für die professionelle Schmerztherapie erarbeitet, um dann gemeinsam die Schaffung der Zusatzbezeichnung „Algesiologie“ bei der Bundesärztekammer zu beantragen. Im Jahr 1996 
beschloss der Ärztetag die Zusatzbezeichnung „Spezielle Schmerztherapie“.

\section{Qualitätssicherung}

Qualitätssicherung ist ein kontinuierlicher Prozess. Hier waren wir vom Zeitpunkt unserer Gründung an sehr aktiv. Ein wichtiges Instrument der Qualitätssicherung ist die Erstellung von Leitlinien. Einerseits initiiert die DGSS eigene Leitlinienprojekte, andererseits vertritt sie die Schmerzmedizin in vielen interdisziplinären Leitlinien.

Schon früh wurde mit Schmerztagebüchern die erste Schmerzdokumentation entwickelt, die Entwicklung des Schmerzfragebogens zusammen mit dem STK stellte einen weiteren Meilenstein dar. Inzwischen sind wir viele Schritte weiter. Mit „Qualitätssicherung in der Schmerztherapie" (QUAST) haben wir die größte anonymisierte Schmerzdatenbank ins Leben gerufen. Die Erkenntnisse aus QUAST sind auch in die Entwicklung der Kerndokumentation zur Qualität in der Schmerztherapie (KeDoQS) eingeflossen - dem zurzeit größten und ambitioniertesten Projekt zur Qualitätssicherung in der Schmerzmedizin in Deutschland.

\section{Nachwuchsförderung}

Um die Weiterentwicklung der Schmerztherapie auch in Zukunft zu gewährleisten, ist es wichtig, dass junge Mediziner weiter forschen und die Schmerzmedizin voranbringen. Deshalb fördern wir den wissenschaftlichen Nachwuchs, indem wir jährlich Promotionsstipendien ausschreiben und unsere Juniorakademie veranstalten. Dort kann sich unser Nachwuchs im Kreise junger Kollegen über Forschungsideen in allen Entwicklungsstadien austauschen.

Zukünftig werden wir besonders förderungswürdigen Projekten in der Grundlagenforschung und in der patientenorientierten Forschung einen deutlich größeren Raum in der öffentlichen Wahrnehmung verschaffen, indem wir Preise ausschreiben, die auf der Eröffnungsveranstaltung des Deutschen Schmerzkongresses verliehen werden, zusammen mit dem Förderpreis für Schmerzforschung und Schmerztherapie. Von Grünenthal gestif- tet wird dieser Preis jedes Jahr im Rahmen des Schmerzkongresses verliehen und hat sich zu dem Deutschen Schmerzpreis entwickelt. Diese Stellung soll zukünftig noch stärker betont werden.

\section{Politische Arbeit}

Um nachhaltige Fortschritte in der Schmerzmedizin durchzusetzen, sind auch strukturelle Veränderungen notwendig, die wir nur erreichen können, wenn wir uns auch politisch engagieren. Dazu haben wir bereits in der Vergangenheit regelmäßig unsere Forderungen öffentlich vorgetragen und publiziert, z. B. in der Ethik-Charta. Diese stellt nicht nur eine ethische Orientierung für die Patientenbetreuung dar. Darin haben wir auch unsere Forderungen formuliert, z. B. nach einer Aufwertung der Schmerzforschung, nach strukturellen Veränderungen in Krankenhäusern oder nach flächendeckenden Strukturen zur Versorgung von Schmerzpatienten. Einen Schritt weiter ist mein Vorgänger Prof. Dr. Rolf-Detlef Treede gegangen. Er forderte und formulierte einen Nationalen Aktionsplan gegen den Schmerz, der in 4 Schritten zu einer besseren Schmerzbekämpfung beitragen soll.

Interessanterweise nahm bereits im Jahr 2002 unser damaliger Bundespräsident Johannes Rau das Thema Schmerz auf seine Agenda. Nicht nur beim damaligen Schmerzkongress setzte er sich für die Ziele der DGSS ein, bereits im Jahr zuvor forderte er:

Wir können und wir müssen viel mehr als bisher für die Schmerztherapie tun. Das ist ein Feld, das lange Zeit sträflich vernachlässigt worden ist. Ich wünsche mir, dass Deutschland bei der Schmerzforschung und bei der Schmerztherapie so schnell wie möglich vorbildlich wird. Das ist nun wirklich zutiefst human und ist im Interesse eines jeden von uns.

Leider ist es bisher vielfach bei Lippenbekenntnissen geblieben.

\section{Öffentlichkeitsarbeit}

Öffentlichkeitsarbeit war bisher nicht der Schwerpunkt unserer Arbeit. Doch auch hier haben wir erste Anstrengungen unternommen. So gingen wir mit der Kampagne "Wehr Dich gegen den Schmerz" im Jahr 2001 - gemeinsam mit verschiedenen Partnern - erstmals an die breite Öffentlichkeit. Die Botschaft lautete damals: Niemand muss chronische Schmerzen ertragen. Auch heute informiert unsere Pressestelle die Öffentlichkeit regelmäBig über Neuigkeiten aus der Schmerzmedizin. In gemeinsamen Initiativen mit Partnern aus anderen Fachgesellschaften, Patienteninitiativen und der Industrie wie „Koalition gegen den Schmerz", „Wege aus dem Schmerz" oder den jährlich stattfindenden "Zukunftsworkshops“ werden aktuelle Themen thematisiert und medial aufbereitet. Doch gerade in diesem Bereich können und wollen wir noch aktiver werden.

Zusammenfassend können wir, wie ich glaube, mit Fug und Recht behaupten, dass wir kontinuierlich an der Verbesserung der Schmerzmedizin in Deutschland gearbeitet und dabei sehr viel erreicht haben. Dass ein Patient mit chronischen Rückenschmerzen heute von einem interprofessionellen Team behandelt wird, wäre vor nicht allzu langer Zeit undenkbar gewesen. Heute entspricht ein solches Vorgehen den Leitlinien. Doch leider gibt es noch immer viel $\mathrm{zu}$ viele Patienten, die schmerztherapeutisch unzureichend versorgt sind. Und es gibt immer noch Ärzte, die sich in ihrer Aus- und Weiterbildung zu wenig mit Schmerzdiagnostik und -therapie auseinandersetzen können. Die Gesellschaft wird also auch weiterhin daran arbeiten müssen, die Voraussetzungen für eine zufriedenstellende Schmerzmedizin zu verbessern. In vielen Bereichen sind wir dafür hervorragend gewappnet.

Unsere Aufgabe ist - das haben wir bereits in der Satzung festgelegt - die interdisziplinäre Kooperation auf dem Gebiet der Schmerzforschung, -diagnostik und -therapie. Dabei lag der Fokus der Gesellschaft in den vergangenen Jahren vornehmlich auf dem Gebiet der Forschung. Damit letztendlich Patienten von den Forschungsergebnissen profitieren können, ist es essenziell, die wissenschaftlichen Erkenntnisse in die klinische Praxis zu bringen. Diesen Transfer gewährleistet die Gesellschaft durch ihre intensi- 
ve Mitarbeit an der Erstellung von Leitlinien und Empfehlungen sowie durch die Entwicklung von Maßnahmen zur Qualitätssicherung. Auch das kontinuierliche Engagement für eine qualifizierte Ausbildung der an der Schmerzmedizin beteiligten Berufsgruppen sorgt dafür, dass neue Erkenntnisse an die Basis transportiert werden. Maßnahmen der Versorgungsforschung schließen den Kreis dieser kontinuierlichen Verbesserung der Schmerztherapie.

\section{Zukunftsvisionen}

Wohin führt uns nun der weitere Weg? Wir haben Enormes geleistet, aber wir sind für die Zukunft noch nicht gut genug aufgestellt. Nach wie vor werden wir in der Öffentlichkeit oft als eine wissenschaftliche Fachgesellschaft ohne Praxisbezug angesehen. Dass wir uns in Leitlinienprojekten und durch Qualitätssicherungsmaßnahmen für eine kontinuierliche Verbesserung der Schmerztherapie engagieren, wissen oft nur Insider. Diese Wahrnehmung werden wir ändern.

Um langfristig die Schmerzmedizin in Deutschland zu verbessern, dürfen wir einzelne Bereiche nicht länger vernachlässigen. Also haben wir uns entschieden, die Schmerzmedizin in Deutschland ab sofort noch aktiver voranzutreiben und alle betroffenen Bereiche miteinander zu vernetzen. Wir wollen den Kontakt zu niedergelassenen Ärzten, Pflegenden und Studenten intensivieren, und so auch neue Mitglieder gewinnen. Wir wollen uns zu einer Schmerzgesellschaft entwickeln, die für alle an der Schmerzmedizin beteiligten Berufsgruppen - Ärzte, Psychologen, Pflegende und Physiotherapeuten, vor allem aber auch Studierende - interessant wird. Wir sind davon überzeugt, dass wir mit unserem Wissen und unseren Projekten für alle in der Schmerzmedizin Tätigen - und das fängt schon bei den Studierenden und Auszubildenden an - attraktive Angebote entwickeln können, sodass sich eine Mitgliedschaft oder sogar eine Mitarbeit in der Gesellschaft lohnt.

Und wir wollen auch mehr als bisher politisch mitreden, denn auch auf politischer Ebene sind Änderungen notwendig, um die Schmerztherapie in Deutschland weiter zu verbessern. Auch die Bundesre- gierung muss das Thema auf ihrer Agenda haben, um das öffentliche Bewusstsein für Schmerzerkrankungen $\mathrm{zu}$ fördern. Nur so wird die Bereitschaft entstehen, öffentliche Forschungsmittel zur Verfügung zu stellen, um das Wissen über die Entstehung und Behandlung von Schmerzen voranzutreiben. Nicht zuletzt wollen wir zukünftig auch stärker auf Patienten zugehen, indem wir sie über die Möglichkeiten der Schmerztherapie informieren und ihnen als kompetente Ansprechpartner zur Verfügung stehen.

Diese notwendigen Veränderungen hat bereits mein Vorgänger Prof. Treede im Nationalen Aktionsplan gegen den Schmerz formuliert. Die darin enthaltenen vier Punkte „Bewusstsein schaffen“, „Forschung fördern“, „Aus- und Weiterbildung verbessern“ und „Versorgung verbessern" sollen ab sofort die Agenda unserer Bestrebungen darstellen.

Wir starten mit der Aufgabe „Bewusstsein schaffen“. Das soll das Motto im ersten Jahr unserer Neuausrichtung sein. Denn gesellschaftliche Veränderungen können nur dann geschehen, wenn Missstände bekannt und im Bewusstsein der Öffentlichkeit verankert sind. Wir wollen erreichen, dass jeder Mensch in Deutschland weiß, was bei Schmerzen zu tun ist, an wen er sich wenden kann, dass akute Schmerzen ausreichend behandelt werden müssen, um chronische Schmerzen zu verhindern, und dass niemand Schmerzen ertragen muss. Daher planen wir, die Aufklärungsaktivitäten zum Thema Schmerz zu verstärken. Als Vorbilder dienen uns dabei Kampagnen von Gesellschaften in anderen Indikationsbereichen, die das Wissen und Denken über diese Erkrankungen in den vergangenen Jahrzehnten massiv verändert haben. Herausragende Beispiele dafür sind Krebs und Aids.

\section{Fortbildungsakademie}

Im nächsten Schritt werden wir unsere Bemühungen in der Aus- und Weiterbildung verstärken. In naher Zukunft soll es daher eine eigene Fortbildungsakademie geben. Diese Akademie wird die Aufgabe haben, Ärzte, Psychologen, Pflegepersonal und die anderen in der Schmerzmedizin tätigen Berufe in der speziellen
Schmerztherapie fortzubilden. Das kann in Form von Präsenzveranstaltungen geschehen, aber auch über unsere Website. Dort werden wir z. B. in Zukunft digitale zertifizierte Fortbildungen anbieten.

\section{Organisatorische Änderungen}

Um all diese Aktivitäten realisieren $\mathrm{zu}$ können, werden wir uns personell verstärken. Ab 2012 soll ein Geschäftsführer das Präsidium in Vollzeit unterstützen. Zurzeit wird nach einer Person mit einem geeigneten Profil für diese Aufgabe gesucht. Aufgabe des Geschäftsführers wird es sein, Kooperationen und Kommunikationsaktivitäten anzustoßen und zu lenken.

So wollen wir für größere Aufmerksamkeit in der Öffentlichkeit sorgen, neue Mitglieder gewinnen und die Zusammenarbeit mit Partnern intensivieren. Neben dem im 2-Jahresrhythmus wechselnden Präsidenten soll über diese neue Position eine personelle Kontinuität gewährleistet werden, die für den Aufbau tragfähiger Partnerschaften, aber auch für unsere Wahrnehmung in der Politik und bei den Kostenträgern unerlässlich ist.

Um diese Aktivitäten zielführend umsetzen zu können, sind allerdings nicht nur erweiterte personelle, sondern auch finanzielle Ressourcen notwendig. Allein mit den bisherigen Mitgliedsbeiträgen werden wir die Aufgaben nicht bewältigen können. Eine zentrale Aufgabe des künftigen Geschäftsführers wird es daher sein, weitergehende Kooperationen mit der pharmazeutischen Industrie $\mathrm{zu}$ initiieren. Bisher waren wir in diesem Bereich eher zurückhaltend. Die Kooperationen beschränkten sich in erster Linie auf den Deutschen Schmerzkongress. Auch hier machen uns Gesellschaften in anderen Bereichen vor, wie man - für beide Seiten fruchtbar - zusammenarbeiten kann, ohne die Neutralität einer wissenschaftlichen Fachgesellschaft zu verlieren.

\section{Partnerschaften}

Eine Idee, die wir hier verfolgen, betrifft die Deutsche Schmerzstiftung. Stiftungszweck der Deutschen Schmerzstiftung soll zukünftig der Nationale Aktionsplan gegen den Schmerz sein. So würde poten- 
ziellen Geldgebern die Grundlage für ein Engagement bereitet werden.

Auch über die Mitgliederwerbung, in der wir uns künftig verstärkt engagieren werden, wollen wir langfristig zusätzliche Gelder akquirieren. Seit einiger Zeit stagniert die Mitgliederzahl bei rund 3000. Wie schon ausgeführt, denken wir, dass wir vielen weiteren Ärzten, Psychologen und anderen in der Schmerzmedizin tätigen Berufsgruppen Interessantes zu bieten haben. Hier werden wir also die Kommunikationsaktivitäten und Angebote intensivieren, um mittel- bis langfristig die Mitgliederzahl deutlich zu steigern.

\section{Kommunikationsoffensive}

Um die genannten Ziele zu erreichen, haben wir gemeinsam mit einer Kommunikationsagentur bereits einen Auftritt erarbeitet, der einerseits modern und fortschrittlich wirkt, andererseits aber eine klare Wiedererkennung gewährleistet. Es wird also ein neues Logo geben, und auch die Website wird neu gestaltet. Nach wie vor - das zeigt auch das neue Logo - steht der Mensch im Mittelpunkt unseres Tuns. Die neue Website, die in rund 4 Wochen online geht, soll insgesamt übersichtlicher werden, sodass Inhalte leichter gefunden werden; zudem soll sie gestrafft werden. Erstmals wird es einen eigenen Bereich für Patienten geben. Gleichzeitig greift der neue Auftritt unsere Vernetzung auf, die sowohl national als auch international zentral für unsere Weiterentwicklung sein wird.

Um diesem Aufbruch in eine neue Zeit Ausdruck zu verleihen, haben wir auf der Mitgliederversammlung die Namensänderung in „Deutsche Schmerzgesellschaft" beschlossen - dieser Name, den wir in den vergangenen Jahren bereits als Appendix geführt haben, drückt genau die Richtung aus, in die wir gehen wollen. Wir sind nicht mehr allein auf die Wissenschaft und das Studium fokussiert, sondern bemühen uns auch um die Schmerztherapie in der täglichen Praxis. Unter dem Motto „Das große Schmerznetzwerk" wollen wir politisch mitreden und die Öffentlichkeit in unsere Arbeit einbeziehen.

Ich bin sicher, dass wir damit für die Gesellschaft und auch für die Schmerz- therapie in Deutschland die richtigen Weichen stellen, und freue mich auf diesen gemeinsamen Weg mit Ihnen!

Für das Präsidium der Deutschen Schmerzgesellschaft

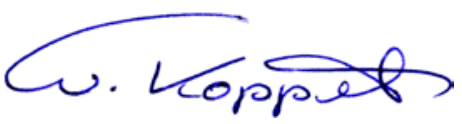

W. Koppert

\section{Korrespondenzadresse \\ Prof. Dr. W. Koppert}

Klinik für Anästhesiologie und Intensivmedizin, Medizinische Hochschule Hannover Carl-Neuberg-Str. 1, 30625 Hannover koppert.wolfgang@mh-hannover.de

\section{Bei langer Krankheit droht Ärzten Versicherungslücke}

Viele Ärzte, die eine Krankentagegeld- und eine Berufsunfähigkeitsversicherung abgeschlossen haben, gehen davon aus, dass sie bei einer schweren Erkrankung zumindest finanziell auf der sicheren Seite sind. Das kann sich aber als Irrtum herausstellen.

In den meisten Fällen ist das Krankentagegeld höher als die Berufsunfähigkeitsdeckung. Das verleitet dazu, den Übergang in die Berufsunfähigkeitsversicherung hinauszuzögern. Die beiden Deckungen greifen jedoch nicht immer nahtlos ineinander. Das Problem: Krankentagegeldversicherungen dienen als Schutz bei einer vorübergehenden Arbeitsunfähigkeit. Die Unternehmen stellen die Leistungen ein, wenn klar ist, dass der Kunde dauerhaft nicht mehr in seinem Beruf arbeiten kann. Meldet sich der Versicherte erst dann beim Berufsunfähigkeitsversicherer oder beim Versorgungswerk, können einige Monate vergehen, bevor die erste Rentenzahlung auf dem Konto eingeht.

Ärzte, die schon länger schwer krank sind sollten daher vorsorglich Leistungen aus der Berufsunfähigkeitsversicherung beantragen. Wenn sich die gesundheitliche Situation bessert, kann man den Antrag problemlos zurückziehen.

Bei den berufsständischen Versorgungswerken ist die Einstufung „berufsunfähig“ nicht immer einfach. Denn die Definition, wann ein Arzt seinen Beruf noch ausüben kann, ist weit gefasst. Ärzte, die zusätzlich eine private Berufsunfähigkeitsversicherung abgeschlossen haben, sollten abklären, ob der Berufsunfähigkeitsschutz auf die konkrete zuletzt ausgeübte Tätigkeit abzielt. Es macht einen Unterschied, ob jemand nicht mehr als Arzt tätig sein kann oder nicht mehr in seiner Spezialisierung. Auch sollten sich Ärzte danach erkundigen, ob sie eine Gruppenversicherung für die Berufsunfähigkeit abschließen können. Dort sind die Bedingungen zum Teil besser und auf die spezifischen Bedürfnisse der Klientel abgestimmt.

Quelle: Ärztezeitung, www.aerztezeitung.de 